Kahan, T., Sellinger, J. J., \& Broman-Fulks, J. J. (2006). Associative and phonological priming effects following letter search on the prime. American Journal of Psychology, 119(2): 239-254. (Summer 2006) Published by the University of Illinois Press (ISSN: 1939-8298).

\title{
Associative and phonological priming effects after letter search on the prime
}

Todd A. Kahan, John J. Sellinger, and Joshua J. Broman-Fulks

\begin{abstract}
Responses to target words typically are faster and more accurate after associatively related primes (e.g., "orange-juice") than after unrelated primes (e.g., "gluejuice"). This priming effect has been used as an index of semantic activation, and its elimination often is cited as evidence against semantic access. When participants are asked to perform a letter search on the prime, associative priming typically is eliminated, but repetition and morphological priming remain. It is possible that priming survives letter search when it arises from activity in codes that are represented before semantics. This experiment examined associative and phonological priming to determine whether priming from phonologically related rhymes would remain after letter search (e.g., "moose-juice"; rhyming items were orthographically dissimilar). When participants read the primes, equivalent associative and phonological priming effects were obtained; both effects were eliminated after letter search. The impact of letter search on semantic and phonological access and implications for the structural arrangement oflexical and semantic memory are discussed.
\end{abstract}


Priming, the speeded response to a target after a related or identical prime relative to an unrelated prime, is of general psychological importance and has been examined using a variety of experimental tasks with widely varying timing parameters. In the single-word semantic priming literature (see Neely, 1991, for a review) the prime and target often are presented within a few hundred milliseconds of one another, whereas the prime and target can be presented minutes apart in word stem completion tasks (see Kinoshita, 2001, for a review). In some instances this priming effect may reflect exclusively (or in part) prospective processing, whereby presentation of the prime causes the target to become preactivated, lessening the critical amount of activation needed for the target to reach threshold. This might occur, for example, if related items were stored nearer to one another in a semantic network (or were connected via stronger links) and activation were to spread throughout this network upon presentation of the prime (Collins \& Loftus, 1975). In other instances priming might reflect exclusively (or in part) retrospective processing, whereby presentation of the target causes the prime to become reactivated; here it is the remembrance of the prime that causes priming. Retrospective priming also depends on the architectural arrangement of words in lexical and semantic memory, but rather than priming signifying the forward-acting spread of activation, priming might reflect retrospective memory processes (Kinoshita, 2001; Neely \& Keefe, 1989; Neely, Keefe, \& Ross, 1989). In addition, priming might reflect a combination of automatic (in the sense that it occurs involuntarily) and controlled processes, regardless of whether it was produced by largely prospective or retrospective mechanisms (see Neely, 1991, and Kinoshita, 2001). Irrespective of the mechanisms responsible for priming (whether it be forward acting or retrospective, automatic or controlled), results from a diverse range of experiments have provided clues regarding the structural arrangement oflexical and semantic memory. The current experiment used the single-word semantic priming paradigm (originated by Neely, 1976). In this task a person responds to a target (typically lexical decision or pronunciation) after silently reading a prime word. Priming is the finding that target responses are faster after related than after unrelated or neutral primes. Prime-target pairs can be related by meaning (e.g., "liquid-:iuice"; semantic priming), association (e.g., "orange-:iuice"; associative priming), or sound (e.g., "moose-juice"; phonological priming), to name just a few.

Although associative and semantic priming are quite robust, they can be reduced or eliminated when participants search the prime for a letter (a procedure originated by Smith, 1979; see Maxfield, 1997, for a review). The removal of priming with this attentional manipulation, called the prime task effect, has been credited as evidence of the blockage of semantic activation (Besner \& MacNevin, 2002; Brown, Roberts, \& Besner, 2001; Chiappe, Smith, \& Besner, 1996; Friedrich, 1993; Friedrich, Henik, \& Tzelgov, 1991; Henik, Friedrich, \& Kellogg, 1983; Henik, Friedrich, Tzelgov, \& Tramer, 1994; Smith, 1979; Smith \& Besner, 2001; Smith, Besner, \& Miyoshi, 1994; Smith, Bentin, \& Spalek, 2001; Smith, Meiran, \& Besner, 2000; Smith, Theodor, \& Franklin, 1983; Stolz \& Besner, 1996, 1998,1999), but see MaTi-Beffa and colleagues for an alternative inhibitory 
view (MaTi-Beffa, Fuentes, Catena, \& Houghton, 2000; Mari-Beffa, Houghton, Estevez, \& Fuentes, 2000).

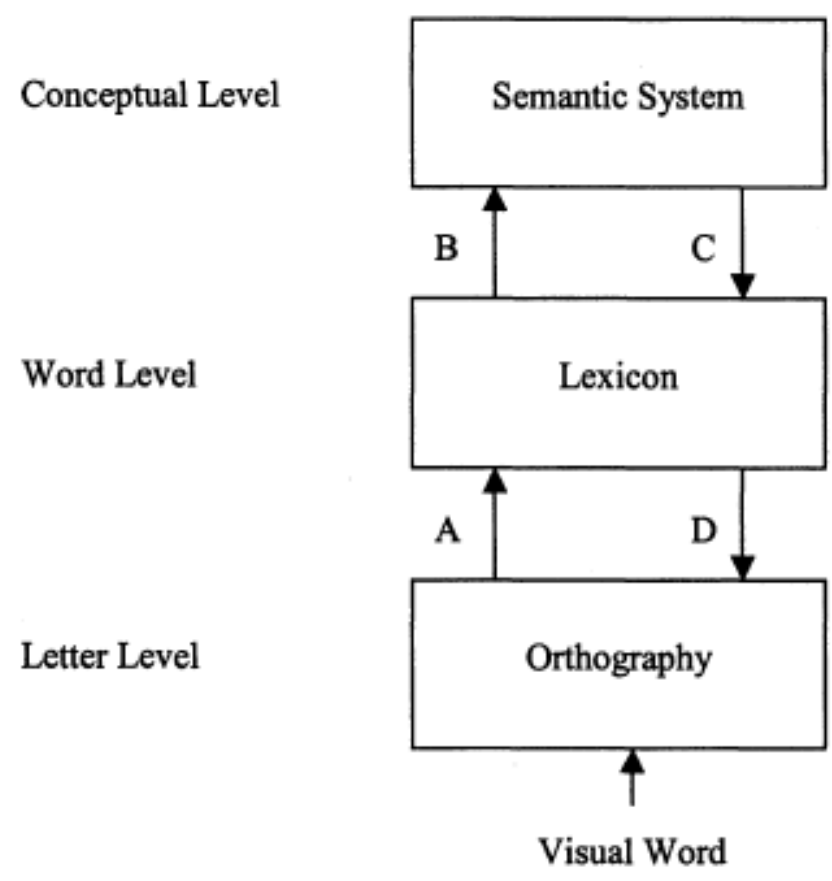

Figure 1. Interactive activation framework

One common explanation for the prime task effect is based on a modified version of McClelland and Rumelhart's (1981) interactive activation framework, depicted in Figure 1 (Brown et al., 2001; Stolz \& Besner, 1996, 1998, 1999; Smith et al. 2000, 2001; alternative explanations for the prime task effect will be addressed in the General Discussion). When a prime word is presented, it is processed in levels (or stages); first letters are extracted from the word, then its lexical entry is retrieved, and finally its meaning is recovered (i.e., semantic activation). In versions of this model that have been used to explain the prime task effect, facilitatory connections are found between layers, whereas inhibitory connections, useful in ensuring that competitors are not retrieved, are found within layers. To explain semantic and associative priming, Stolz and Besner (1996) contended that words in the mep.tallexicon are linked to their own meanings in the semantic system as well as the meanings of related words (see also Ferrand \& New, 2004, for a detailed discussion of pure semantic and associative priming in this framework). Semantic and associative priming effects result from between-level spreading activation. When a word is presented as the prime (e.g., "student"), activation from letters facilitates a lexical representation (via Pathway $A$ ), and this in turn activates the meaning of the word presented along with semantic and associatively related words (via Pathway B). If a related word (e.g., "teacher") is then presented as the target, less bottom-up activation is needed for this target's meaning 
to reach threshold, and target responding is faster than with an unrelated target, assuming within-level semantic inhibition does not eliminate the activation that was transmitted via Pathway B. Stolz, Robidoux, and Besner (2000) successfully simulated semantic priming using this framework. In addition, this facilitation may be strengthened with top-down activation of the related word's lexical entry (via Pathway C), further decreasing response latencies relative to those of unrelated words that will not benefit from preactivation. This can be envisioned as occurring in either a localist or distributed manner. Although the default is to process text for meaning, letter search may focus resources at early stages of processing, blocking activation from reaching the semantic system (i.e., Pathway $B$ is blocked by letter search).

This account helps researchers explain two significant dissociations. Letter search eliminates semantic and associative priming, but it does not eliminate repetition priming (Friedrich et al., 1991) or morphological priming (Stolz \& Besner, 1998). Ifletter search blocks Pathway B in Figure 1, semantic and associative priming will be eliminated but repetition priming will remain because of preactivation at the letter and word levels. Likewise, letter search does not eliminate morphological priming (e.g., "marked" as a prime for "mark") (Stolz \& Besner, 1998, Experiment 2). These data suggest that morphology is coded in a layer before semantic analysis; consequently, preactivation of morphological codes was not blocked by letter search on the prime, as it was for semantic codes. One possibility is that the morpheme is the unit represented in the lexicon, and both results (preserved repetition and morphological priming after letter search on the prime) reflect continued activation at the lexical level (Stolz \& Besner, 1998). It is also possible that preserved repetition priming after letter search reflects (or partly reflects) the activation of phonological codes, which are not blocked by letter search.

The current experiment examined priming for items that were related by association or sound. The associatively related items that were used herein were not all pure in the sense that some of these items had similar meanings (e.g., "sneaker-shoe"), whereas others did not (e.g., "orange-juice"); all items used were strong associates. The phonologically related items that were used did not overlap in meaning, association, or orthography (e.g., "moose-juice"). Hereafter, associative relatedness is used as a generic term to describe items that were associatively related and might also overlap semantically. The current experiment had two aims. First, we sought to determine whether associative but not phonological priming would be eliminated after letter search. The answer to this question has implications for models of lexical and semantic memory. If letter search works to block semantic level activation only, performing a letter search on the prime should not affect priming for codes represented at levels earlier than semantics. In the modified interactive activation network (Stolz \& Besner, 1996), associative priming depends on activation in the semantic system, whereas phonological priming probably is activated and represented before semantics (Tan, Hoosain, \& Siok, 1996); therefore, phonological priming but not associative priming might remain after letter search. The second 
aim was to provide a direct comparison between associative priming (e.g., "orange" as a prime for 'juice") and phonological priming (e.g., "moose" as a prime for 'juice") using the same target words and orthographically dissimilar items. Although priming from words that share associative or semantic overlap is well documented in lexical decision literature (Hutchison, 2003; Lucas, 2000; Neely, 1991), the results are mixed with regard to pure phonological priming. Hillinger (1980) obtained priming for phonologically related rhymes in lexical decision tasks, but Martin and Jensen (1988) failed to replicate this effect. More recently Rouibah, Tiberghien, and Lupker (1999) obtained phonological priming from masked primes in a number of tasks including color matching, semantic matching, and phonological matching. Rouibah, Tiberghien, and Lupker did not examine performance in a lexical decision task. It should be noted that because masked rather than clear primes were used, the priming that was reported might reflect a different mechanism (perhaps more automatic in nature). Irrespective of whether the mechanism responsible for priming in any given task is forward or backward acting, automatic or controlled, these effects probably reflect the same underlying structural configuration, and it is this architecture that is of primary interest here.

This experiment was not intended as a test of Stolz and Besner's (1998) semantic-level blocking account because blocking semantic-level activation does not preclude blocking elsewhere in the network. If associative but not phonological priming is eliminated with letter search, our results will suggest that repetition and morphological priming (after letter search) reflect activation in the lexicon (or phonological codes that mayor may not be stored in the lexicon). However, ifboth associative and phonological priming are eliminated after letter search on the prime, our results will be consistent with the views that continued repetition and morphological priming after letter search (as reported by Friedrich et al., 1991, and Stolz \& Besner, 1998) reflect activity in orthographic or morphological codes, rather than phonological codes, which are stored separately in the network, and that letter search blocks the mechanism responsible for priming to both the semantic system and phonology.

\section{EXPERIMENT}

\section{METHOD}

\section{Participants}

One hundred forty-four students participated for extra credit in an introductory psychology course; 96 were from the University of Southern Mississippi, and 48 were from Bates College. Half of the students from each school participated in each prime task. All participants were native English speakers with normal or corrected-to-normal vision. 


\section{Materials}

The experiment was composed of 240 trials, 120 of which had word targets and 120 nonword targets. Words were used as primes throughout the experiment. Of the trials with word targets, 40 were phonologically related rhymes (e.g., "moose-juice") that were orthographically dissimilar, 40 were associatively related (e.g., "orange-juice"), and 40 were unrelated (e.g., "glue-juice"); these were counterbalanced such that every target appeared in every condition across participants. (For example, one third of the participants saw the target word Juice" in the phonological condition, one third saw this word in the associatively related condition, and the remaining third saw this word in the unrelated condition.) Each prime had a letter duplicated above it. In half of the trials the letter above the prime was contained in the prime word (this letter was chosen randomly); in the other half, the letter was not found in the prime word. This too was counterbalanced across participants (e.g., half of the participants who were shown the prime-target pair "moose-juice" saw "e" duplicated above the prime word "moose," the other participants saw "f' duplicated above the prime word). The unrelated trials were created by randomly re-pairing 20 of the associatively related primes and 20 of the phonologically related primes (e.g., "sneaker" from the "sneaker-shoe" pair and "glue" from the "glue-shoe" pair, respectively). Multiple lists were used to balance this; if an unrelated item was created from a phonological prime on one list (e.g., "glue-juice," where "glue" was taken from the pair "glue-shoe"), its associative prime was used to make an unrelated trial on another list (e.g., "sneaker-juice," where "sneaker" was taken from the pair "sneaker-shoe"). Crossing the three relatedness conditions (associative, phonological, or unrelated), two prime response types (yes vs. no), and two manners of creating unrelated items (created from associative vs. phonological primes) yielded 12 experimental lists. The average target length was five letters, and the average frequency was 102 per million words in the English language (KuCera \& Francis, 1967).

We created nonword targets by changing one or two letters in real English words; all targets remained pronounceable. None of the words from which the nonwords were created was related with the prime with which it was paired. These nonword trials, necessary for the lexical decision task, were not counterbalanced and were treated as filler items.

In addition to the 240 experimental trials, 12 practice trials were created. Six of these had word targets, and the remaining six had nonword targets. One third of the word targets were from each relatedness type (phonological, associative, and unrelated). The practice trials were not counterbalanced, none of the words or nonwords in the practice were used in the experimental trials, and data from the practice were discarded.

\section{Procedure}

Participants were randomly assigned to either the silent read group or letter search group. Students in the letter search group were told that their task was to decide whether the letter duplicated above the prime word was contained in that word. The" I?" key was used to indicate a "yes" response, and the "z" key 
was used to indicate a "no" response. Students in the silent read condition were instructed to simply look at the prime; no specific instructions were given regarding the letter that was duplicated above this word. Both groups were instructed to decide whether the target was a correctly spelled English word and to make their responses as quickly and accurately as possible. If it was, they were told to press the "/?" key with their right index finger; otherwise, they were instructed to press the " $z$ " key with their left index finger.

Each trial began with a fixation centered on the computer monitor ("*ᄎ*") for $100 \mathrm{~ms}$. This was followed by a 100-ms blank screen, which in tum was followed by a prime word (e.g., "laugh") that had letters duplicated above it (e.g., "uuuuu"), both in lowercase. The prime was displayed for $300 \mathrm{~ms}$ and was followed by a 1,300 -ms blank screen. During this fixed window of time participants in the letter search group made their letter search response. A second fixation point ("*") then appeared for $100 \mathrm{~ms}$ and was followed by another 100-ms blank screen before the target (e.g., "half') was presented, also in lowercase. The target remained visible until the lexical decision response was made. Mter each trial there was a 2,000-ms blank intertrial interval. The words were shown in a white 12-point Courier font on a black background. The same displays and timing parameters were used for the two groups (letter search and silent read). Presentation of events and the recording of responses were controlled with MEL v.2.0 software (Schneider, 1988) running on $\mathrm{PC}$-compatible microcomputers.

\section{RESULTS}

Reaction times and proportions of correct responses to the target in the different experimental conditions are displayed in Table I. These data were submitted to a 3 (relatedness: phonological, associative, or unrelated) $X 2$ (prime task: silent read or letter search) mixed ANOVA, with relatedness a within-subject variable and prime task a between-subject variable $\left(F_{1}\right)$. The data were also submitted to a 3 (relatedness: phonological, associative, or unrelated) $\times 2$ (prime task: silent read or letter search) within-items ANOVA, with both relatedness and prime task treated as within-items variables $\left(F_{2}\right)$. Only trials with correct prime and target responses are reported, collapsing across schools.! There was a main effect of relatedness, $F_{1}(2,284)=4.79, p<.05, F_{2}(2,238)=3.72, p<.05$, and no main effect of prime task, all ps $>.05$. More importantly, there was an interaction between relatedness and prime task in the subject analysis, $F_{1}(2,284)=4.22, p<.05, F_{2}(2,238)=1.23, p>.05$. To break this interaction down, we conducted ANOVAS on each prime task separately. For silently read primes, equal priming effects were obtained for phonologically $(+32$ $\mathrm{ms})$ and associatively (+34 ms) related targets, $F_{1}(2,142)=7.90, p<.05$, $\mathrm{F}_{2}(2,238)=3.77, p<.05$; see the top portion of Table 1. Mter letter search on the prime, no priming effects were obtained for either phonologically $(-5 \mathrm{~ms})$ or associatively $(+5 \mathrm{~ms})$ related targets, $F_{1}(2,142)=.64, p>.05$, $\mathrm{F}_{2}(2,238)=.53, p>.05$; see the bottom portion of Table 1 . The only effect that reached significance in the accuracy data was a main effect of prime task, $F_{1}(1,142)=5.81, p<.05, F_{2}(1,238)=5.05, p<.05$; responses to the target were more accurate in the silent read condition. 
Table 1. Mean time to respond to the target in milliseconds (and proportion correct) as a function of the prime task and the prime-target relationship, along with priming effects and $95 \%$ confidence intervals

\begin{tabular}{lllcc}
\hline & & \multicolumn{3}{c}{$95 \%$ confidence interval } \\
\cline { 3 - 5 } Prime task & $\begin{array}{l}\text { Prime-target } \\
\text { relationship }\end{array}$ & \multicolumn{1}{c}{ Mean } & Lower & Upper \\
\hline Silent read & Unrelated & $748(.95)$ & $700(.94)$ & $796(.96)$ \\
& Phonological & $716(.95)$ & $675(.93)$ & $757(.96)$ \\
& Priming & $+32^{*}(.00)$ & & \\
& Associative & $714(.96)$ & $673(.95)$ & $754(.97)$ \\
& Priming & $+34^{*}(-.01)$ & & \\
Letter search & Nonword & $908(.94)$ & $843(.92)$ & $973(.95)$ \\
& Unrelated & $702(.93)$ & $670(.91)$ & $734(.95)$ \\
& Phonological & $707(.94)$ & $673(.93)$ & $741(.95)$ \\
& Priming & $-5(-.01)$ & & \\
& Associative & $697(.94)$ & $662(.93)$ & $731(.95)$ \\
& Priming & $+5(-.01)$ & & \\
& Nonword & $829(.91)$ & $786(.90)$ & $872(.93)$ \\
\hline
\end{tabular}

${ }^{*} p<.05$.

\section{DISCUSSION}

The results are clear: Equal priming effects are obtained for phonologically (+32 ms) and associatively (+34 ms) related prime-target pairs after silently read primes in a lexical decision task, and these effects are eliminated with letter search. The data from the silent read condition provide a direct comparison between priming for phonologically related rhymes and associatively related items using the same English target words; results indicate that these priming effects are equal in magnitude. These priming effects may reflect some combination of forward-acting spreading activation and retrospective prime recovery. Notwithstanding the mechanisms involved in producing priming here (strategic or otherwise), these effects provide clues regarding the architecture oflexical and semantic memory. The finding that these priming effects were equivalent helps to demonstrate that we were starting from the same point when trying to eliminate priming with letter search. These results replicate the work of Hillinger (1980), who obtained phonological priming in a lexical decision task, and run counter to that of Martin and Jensen (1988), who failed to find phonological priming in this task. The data from the letter search condition indicate that letter search eliminates both of these priming effects. Therefore, preserved repetition (Friedrich et al., 1991) and morphological (Stolz \& Besner, 1998) priming after letter search must not reflect activity in phonological codes. 


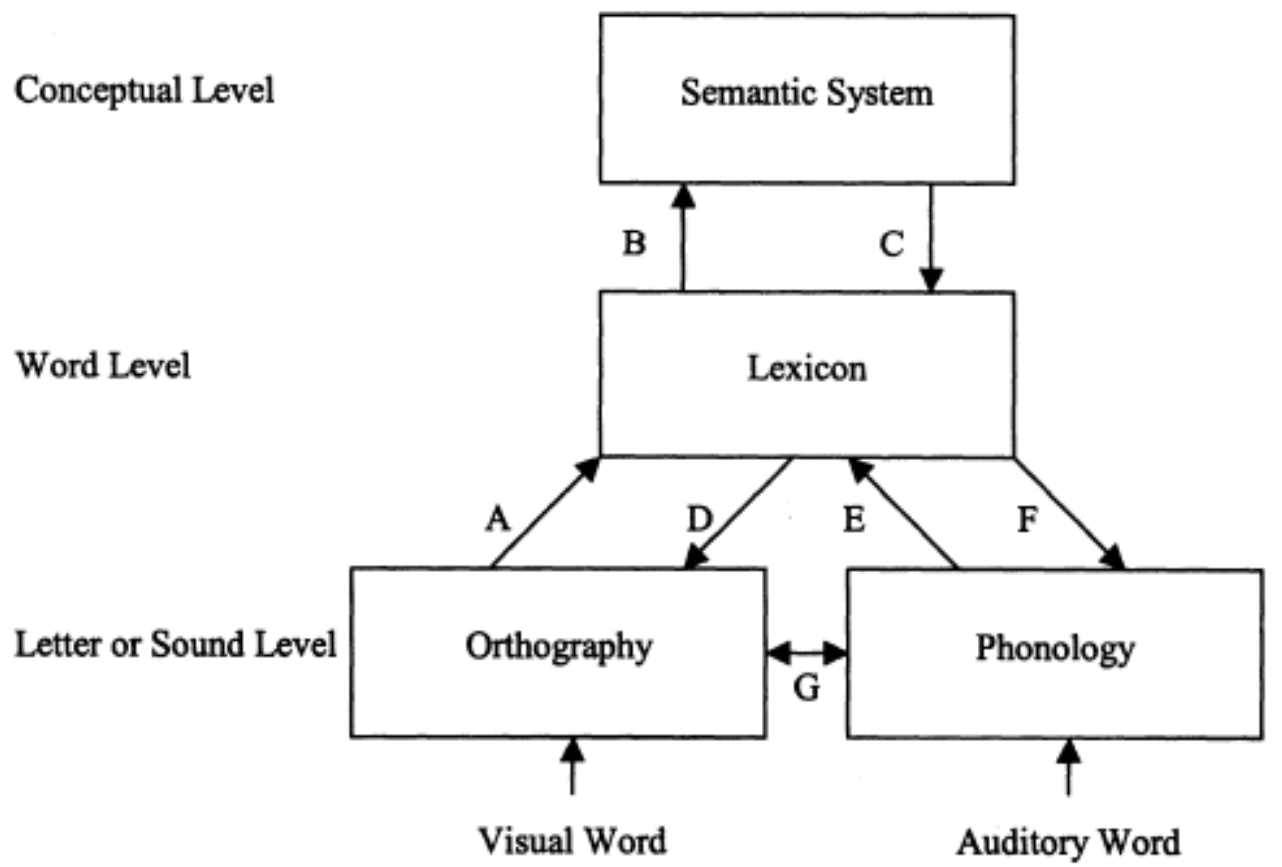

Figure 2. Interactive activation framework with phonology stored in a distinct level from orthography

These data are consistent with the view that letter search results in blockages at several points in the information processing stream. Ifletter search on the prime resulted only in blockages to the semantic level (Pathway B in Figure 1), one might have predicted that phonological priming but not associative priming would survive letter search because the former but not the latter is likely to be represented in a layer before semantics. However, phonological codes might be stored in a layer distinct from orthography, as depicted in Figure 2, and letter search may work to block access to both the semantic (Pathway B) and phonological networks (Pathways F and G), where these pathways represent connections on which not only can forward-acting activation travel but also retrospective and strategic processes may occur. Letter search might focus attention on orthography at the expense of phonology and semantics. This could be envisioned in several ways different from what is depicted in Figure 2. For example, although Figure 2 portrays phonological codes as becoming activated after orthography for visually presented words, it is certainly possible that orthography and phonology become activated in parallel (i.e., a direct connection from visual words to phonological codes might exist), and letter search may block this pathway along with pathways $B, F$, and $G$ in Figure 2. Alternatively, phonological codes may be represented in a layer after semantic codes (for visually presented English words), although this would contradict the findings of Tan et ai. (1996) with Chinese characters. What is clear is that phonological codes probably are stored separately from orthography and morphology. Because repetition priming (Friedrich et al., 1991) and morphological priming (Stolz \& Besner, 1998) survive letter search on the prime, one would expect that phonological priming 
would also remain after letter search if phonological codes were represented in the same network as orthography and morphology. This did not occur, and therefore our results run counter to the spreading activation model proposed by Collins and Loftus (1975). This model has two parallellayers (or networks). Phonology and orthography are represented in the same lexical network, apart from the semantic network.

Ifletter search prevents phonological activation altogether, this would undermine the prevalent view that visually presented words always activate their phonological representations (Frost, 1998; Perfetti \& Bell, 1991). Besner and Care (2003) recently developed a clever technique (the task choice procedure) for assessing whether phonological codes are involuntarily extracted from text. Participants were given a target nonword and were asked to either pronounce it aloud or make a case decision (pressing one button for uppercase and another for lowercase); a cue indicated which task was to be performed. This cue either preceded the target by 750 ms or appeared simultaneously with the target, and the target was either visually degraded or presented clearly. Besner and Care reasoned that when the task is known in advance (i.e., at the -750-ms stimulus onset asynchrony [SOA]) participants ought to respond more slowly to degraded than to clearly presented targets. Furthermore, if participants processed the target spontaneously, converting graphemes to phonemes effortlessly, any effect of target contrast ought to be absorbed into the time necessary to decode the cue when the target and cue are presented simultaneously (i.e., at the O-ms SOA). Therefore, if an interaction were found between target contrast and SOA, such that the contrast effect emerged when the task was known in advance (at the -750-ms SOA) but not when the task cue was given simultaneously with the target (at the O-ms SOA), results would suggest that phonology was extracted in parallel with deciphering of the cue at the O-ms SOA. This was not found. Rather, these two variables (SOA and contrast) had additive effects. These results are best explained as arising because target processing followed cue decoding; the extraction of phonological codes was delayed until after the task was known.

Alternatively, letter search may not have blocked activation of phonological codes altogether but may have slowed it. Perhaps semantic and phonological codes were activated in the same manner as during the silent read condition, but activation of these codes occurred too late to effectively speed target responses. However, it seems doubtful that prime letter search merely slows processing because this cannot explain all of the extant data. Specifically, it is not clear why access to some codes (e.g., semantic and phonological) would be slowed whereas access to other codes (e.g., morphological; see Stolz \& Besner, 1998) would proceed at full speed after letter search.[2]

Another possibility, in addition to blocking and slowing, is that the prime word is inhibited when attention is focused at the letter level (MariBeffa, Fuentes, et al., 2000; Mari-Beffa, Houghton, et al., 2000). It has been well established that responses to the color of a printed color word (as in Stroop, 1935) are slower when that color had previously been ignored than when that color had not previously been ignored (Neill, 1977). Thisnegative 
priming effect may reflect an inhibitory mechanism that reduces the activation of ignored words (Tipper, 1985). Similarly, by focusing attention at the letter level, inhibitory mechanisms may have worked in opposition to facilitatory mechanisms, resulting in the elimination of priming. Perhaps semantic and phonological codes were activated, but letter search initiated an inhibitory mechanism that wiped out priming. However, as was the case with the slowing explanation, this inhibitory account cannot explain why repetition (Friedrich et at, 1991) and morphological (Stolz \& Besner, 1998) priming remain,facilitatory whereas only phonological and associative codes are inhibited after letter search.

If priming in this experiment reflects the automatic spread of activation through lexical and semantic memory, then the elimination of priming might be seen as problematic for theories that contend that semantic and phonological codes are obligatorily activated when a word is seen (see Neely \& Kahan, 2001, for a discussion of automatic semantic activation). However, priming can be eliminated for a number of reasons, including blocking, slowing, and inhibiting activation in the lexical and semantic system. Only the blocking explanation, which more easily accounts for the extant data, poses threats to the claim that semantic and phonological activation always occur, if one assumes that these priming effects reflect spreading activation. (See Mathis, 2002, for a more detailed discussion of blocking, slowing, and inhibition accounts as they relate to object recognition.)

However, with any of these explanations it is important to remember that semantic and phonological activation are not measured directly. Rather, activation is inferred from positively (or negatively) signed priming effects. The elimination of priming often is cited as evidence that semantic (or in this case phonological) activation was prevented, but priming may reflect processes other than spreading activation. In fact, the time between presentation of the prime and target is long in letter search experiments (1,800 ms herein), in comparison with other single-word semantic priming experiments (Neely, 1991) that are attributed to spreading activation, but was short in comparison with other priming tasks. For example, in word stem completion experiments the time between study and test often is several minutes (Kinoshita, 2001). It is possible that associative and phonological priming in the silent read condition arise from a retrospective process, perhaps akin to involuntary aware memory (see Kinoshita, 2001; see also Mace, 2003a, 2003b), and that the elimination of priming with letter search reflects the blockage of this retrospective process rather than the blockage of spreading activation. In the current experiment the target may involuntarily cue the retrieval of the prime episode when the prime is silently read but not when the prime's letters are scrutinized, and this conscious remembrance of the prime (and associated or phonologically related words) may in tum reactivate (or strengthen) target processing. Accordingly, it is the remembrance of the prime that is responsible for the faster responses to related than to unrelated targets. It has been reported that involuntary aware memory produces priming in a number of implicit tasks including word stem completion (Mace, 2003b) and implicit category generation (Mace, 2003a) and that involuntary aware memory depends on semantic processing of the study words; priming from involuntary 
aware memory did not occur when participants were required to count the number of syllables in study words. Similarly, involuntary aware memory may contribute to priming in lexical decision when the prime is silently read but not when a letter search is performed on the prime.

The elimination of priming after letter search on the prime does not occur for repeated prime-target pairs (Friedrich et al., 1991) or morphologically related pairs (Stolz \& Besner, 1998); however, it does occur for phonologically related rhymes and associatively related pairs, which produce equivalent priming when the prime is silently read (current experiment). Theories of priming, whether they are forward acting or retrospective, automatic or controlled, generally rely on the architectural arrangement oflexical and semantic memory to help explain speeded responses to related relative to unrelated words. That priming is eliminated for some prime-target relationships (e.g., associative and phonological) but not others (e.g., repetition and morphological) constrains this structural arrangement. Taken together, our results suggest that repetition and morphological priming after letter search are based on the same representational structures, which are likely to be morphological rather than orthographic (Stolz \& Besner, 1998) or phonological (current experiment), and that letter search may block the mechanism that produces priming to both the semantic system and phonology. Specifically, letter search may block Pathways B, F, and G in Figure 2, where these pathways represent connections on which not only can forward-acting activation travel but also retrospective and strategic processes may occur. These results run counter to the spreading activation model proposed by Collins and Loftus (1975), in which orthographic and phonological codes are represented in the same processing layer and are more easily explained in the modified interactive activation framework proposed by Stolz and Besner (1996).

\section{NOTES}

1. When school was added as a between-subjects (within-items) factor it did not produce a main effect, nor did it interact with the other factors. A pattern of data and significance levels identical to those reported here emerged when we analyzed the data without restricting our analysis to trials with correct prime responses. 2. We thank Jennifer Stolz for bringing this to our attention. 


\section{REFERENCES}

Besner, D., \& Care, S. (2003). A paradigm for exploring what the mind does while deciding what it should do. Canadian Journal of Experimental Psychology, 57, 311-320.

Besner, D., \& MacNevin, C. (2002). When are morphemic and semantic priming observed in visual word recognition? CanadianJournalofExperimentalPsychology, 56, 112-119.

Brown, M. S., Roberts, M. A, \& Besner, D. (2001). Semantic processing in visual word recognition: Activation blocking and domain specificity. Psychonomic Bulletin \& Review, 8,778-784.

Chiappe, P. R., Smith, M. C., \& Besner, D. (1996). Semantic priming in visual word recognition: Activation blocking and domains of processing. Psychonomic Bulletin \& Review, 3, 249-253.

Collins, AM., \& Loftus, E. F. (1975). A spreading-activation theory of semantic processing. Psychological Review, 82, 407-428.

Ferrand, L., \& New, B. (2004). Semantic and associative priming in the mental lexicon. In P. Bonin (Ed.), Mental lexicon: Some words to talA about words (pp. 25-43). Hauppauge, NY: Nova Science Publisher.

Friedrich, F. j. (1993, November). Prime task effects on naming RTs. Paper presented at the 34th annual meeting of the Psychonomic Society, Washington, DC.

Friedrich, F.j., Henik, A, \& Tzelgov,J. (1991). Automatic processes in lexical access and spreading activation. Journal of Experimental Psychology: Human Perception and Perjrmnance, 17, 792-806.

Frost, R (1998). Toward a strong phonological theory of visual word recognition: True issues and false trails. Psychological Bulletin, 123, 71-99.

Henik, A, Friedrich, F.]., \& Kellogg, W. A (1983). The dependence of semantic relatedness effects upon prime processing. Memory \& Cognition, 11, 366-373.

Henik, A, Friedrich, F.]., Tzelgov,]., \& Tramer, S. (1994). Capacity demands of automatic processes in semantic priming. Memory \& Cognition, 11, 366-373.

Hillinger, M. I. (1980). Priming effects with phonemically similar words: The encoding-bias hypothesis reconsidered. Memory \& Cognition, 8, 115-124.

Hutchison, K. A (2003). Is semantic priming due to association strength or featural overlap? A micro-analytic review. Psychonomic Bulletin \& Review, 10, 785-813.

Kinoshita, S. (2001). The role ofinvoluntary aware memory in the implicit stem and fragment completion tasks: A selective review. Psychonomic Bulletin \& Review, 8, 58-69. 
KuCera,]., \& Francis, W. (1967). Computational analysis of present day American English. Providence, RI: Brown University Press.

Lucas, M. (2000). Semantic priming without association: A meta-analytic review. Psychonomic Bulletin \& Review, 7, 618-630.

Mace,]. H. (2003a). Involuntary aware memory enhances priming on a conceptual implicit memory task. American Journal of Psychology, 116, 281-290.

Mace,]. H. (2003b). Study-test awareness can enhance priming on an implicit memory task: Evidence from a word completion task. American Journal of Psychology, 116,257-279.

Mari-Beffa, P., Fuentes, L.]., Catena, A, \& Houghton, G. (2000). Semantic priming in the prime task effect: Evidence of automatic semantic processing of distractors. Memory \& Cognition, 28, 635-647.

Mari-Beffa, P., Houghton, G., Estevez, A F., \& Fuentes, L.]. (2000). Word based grouping affects the prime-task effect on semantic priming. Journal ofExperimental Psychology: Human Perception and Performance, 26, 469-479.

Martin, R C., \&Jensen, C. R (1988). Phonological priming in the lexical decision task: A failure to replicate. Memory \& Cognition, 16, 505-521.

Mathis, K. M. (2002). Semantic interference from objects both in and out of a scene context. Journal of Experimental Psychology: Learning, Memory, and Cognition, 28, 171-182.

Maxfield, L. (1997). Attention and semantic priming: A review of prime task effects. Consciousness and Cognition, 6, 204-218.

McClelland,]. L., \& Rumelhart, D. E. (1981). An interactive activation model of context effects in letter perception: I. An account of basic findings. Psychological Review, 88,375-407.

Neely,]. H. (1976). Semantic priming and retrieval from lexical memory: Evidence for facilitatory and inhibitory processes. Memory \& Cognition, 4, 648-654.

Neely,]. H. (1991). Semantic priming effects in visual word recognition: Aselective review of current findings and theories. In S. Besner \& G. Humphreys (Eds.) , Basic processes in reading: VISual word recognition (pp. 264-336). Hillsdale, 1lij: Erlbaum.

Neely,]. H., \& Kahan, T. A (2001). Is semantic activation automatic? A critical re-evaluation. In H. L. Roediger,]. S. Nairne, I. Neath, \& A M. Surprenant (Eds.), The nature of remembering: Essays in honor of \&bert c. Crowder (pp. 69-93). Washington, DC: American Psychological Association. 
Neely,]. H., \& Keefe, D. E. (1989). Semantic context effects on visual word processing: A hybrid prospective/retrospective processing theory. In G. H. Bower (Ed.), The psychology of learning and motivation: Advances in research and theory (Vol. 24, pp. 207-248). New York: Academic Press.

Neely,]. H., Keefe, D. E., \& Ross, K. (1989). Semantic priming in the lexical decision task: Roles of prospective prime-generated expectancies and retrospective semantic matching. Journal of Experimental Psychology: Learning, Memory, and Cognition, 15,1003-1019.

Neill, W. T. (1977). Inhibitory and facilitatory processes in attention. Journal of Experimental Psychology: Human Perception and Performance, 3, 444-450. Perfetti, C. A., \& Bell, L. (1991). Phonemic activation during the first $40 \mathrm{~ms}$ of word identification: Evidence from backward masking and priming. Journal of Memory and Language, 30, 473-485.

Rouibah, A, Tiberghien, G., \& Lupker, S.]. (1999). Phonological and semantic priming: Evidence for task-independent effects. Memory \& Cognition, 27, 422-437.

Schneider, W. (1988). Micro-experimentallaboratory: An integrated system for IBM PC compatibles. Behavioral Research Methods, Instruments, \& Computers, 20, 206-217.

Smith, M. C. (1979). Contextual facilitation in a letter search task depends on how the prime is processed. Journal of Experimental Psychology: Human Perception and Performance, 5, 239-251.

Smith, M. C., Bentin, S., \& Spalek, T. M. (2001). Attention constraints of semantic activation during visual word recognition. Journal of Experimental Psychology: Learning, Memory, and Cognition, 27, 1289-1298.

Smith, M. C., \& Besner, D. (2001). Modulating semantic feedback in visual word recognition. Psychonomic Bulletin \& Review, 8, 111-117.

Smith, M. C., Besner, D., \& Miyoshi, H. (1994). New limits to automaticity: Context modulated semantic priming. Journal of Experimental Psychology: Learning, Memory, and Cognition, 20, 104-115.

Smith, M. c., Meiran, N., \& Besner, D. (2000). On the interaction between linguistic and pictorial systems in the absence of semantic mediation: Evidence from a priming paradigm. Memory \& Cognition, 28, 204-213.

Smith, M. C., Theodor, L., \& Franklin, P. E. (1983). The relationship between contextual facilitation and depth of processing. Journal of Experimental Psychology: Learning, Memory, and Cognition, 9,697-712.

Stolz,]. A, \& Besner, D. (1996). Role of set in visual word recognition: Activation and activation blocking as nonautomatic processes. Journal of Experimental Psychology: Human Perception and Performance, 5, 1166-1177. 
Stolz,]. A, \& Besner, D. (1998). Levels of representation in visual word recognition: A dissociation between morphological and semantic processing. Journal of Experimental Psychology: Human Perception and Performance, 24, 1642-1655.

Stolz,]. A., \& Besner, D. (1999). On the myth of automatic semantic activation in reading. Current Directions in Psychological Science, 8, 61-65.

Stolz,]. A, Robidoux, S., \& Besner, D. (2000). Semantic priming in the context of an interactive activation framework: Explorations in connectionist modeling. Unpublished manuscript.

Stroop,]. R. (1935). Studies of interference in serial verbal reactions. journal of Experimental Psychology, 18, 643-662.

Tan, L. H., Hoosain, R., \& Siok, W. W. T. (1996). Activation of phonological codes before access to character meaning in written Chinese. journal of Experimental Psychology: Learning, Memory, and Cognition, 22, 865-882.

Tipper, S. P. (1985). The negative priming effect: Inhibitory effects of ignored primes. Quarterly journal of Experimental Psychology, 37A, 571-590. 